\title{
Transferability of cleavage fracture parameters between notched and cracked geometries
}

\author{
C. Bouchet ${ }^{1}$, B. Tanguy ${ }^{1, \star}$, J. Besson $^{1}$, A. Pineau ${ }^{1}$ and S. Bugat ${ }^{2}$ \\ ${ }^{1}$ Centre des Matériaux, ENSMP, UMR CNRS 7633, BP 87, Evry cedex 91003, France \\ ${ }^{2}$ EDF R\&D, Les Renardières, Moret sur Loing cedex 77818, France \\ ^ Corresponding author. Email: Benoit.Tanguy@ensmp.fr
}

\begin{abstract}
The present study investigates the temperature and specimen geometry dependence of cleavage fracture micromechanisms of a French pressure vessel steel (A508 Cl.3) over a temperature range which covers the lower shelf up to the DBT fracture toughness range. Notched tensile (NT) specimens with 3 different radii and CT specimens with two different thicknesses (12.5 and $25 \mathrm{~mm}$ ) were used to study the effect of temperature and geometry (constraint) on (i) the nature of the defects involved in the cleavage triggering, (ii) the mechanical parameters (stress and strain) values (iii) the Weibull parameters. The fractographic investigations showed that Manganese sulfide clusters and/or stringers were involved in the cleavage initiation process on the notched geometry at all temperatures from $-130^{\circ} \mathrm{C}$ whereas they were never encountered in the cracked geometries. It is shown that only NT tests with a mean fracture strain lower than $25 \%$ have to be considered to make sure that the same nature of defects will be involved in the cleavage initiation. The mean value of the local stress was found to be approximately constant for NT and CT25 specimens over the temperature range $\left[-150^{\circ} \mathrm{C} ;-50^{\circ} \mathrm{C}\right]$. Also a unique set of Weibull parameters was found to describe all the NT tests over this temperature range.
\end{abstract}

\section{Introduction}

In the recent past, the local approach to fracture has shown its capacity to be a predictive tool in many complex cases of structural integrity assessment [1,2]. Brittle cleavage fracture of steels is one of the fields where local approach has brought a lot of understanding (e.g. effect of prestraining, effect of dynamic loading). In the nuclear industry, local approach to fracture is often used as a complementary tool to the code (e.g. ASME) in order to reduce the empirical margins and to contribute to a better understanding of the physical mechanisms which can lead to fracture. Most of the successful models devoted to the description of cleavage fracture are based on the weakest link assumption. One of the pioneering models devoted to low temperature cleavage fracture, the Beremin model [3], has linked the fracture probability with the defects population through the Weibull stress concept. Besides its physical bases, the success of this model relies on the small number of parameters that has to be calibrated. In the original Beremin's work, notched tensile specimens with different radii were used to calibrate the parameters over a given range of temperatures; the model was then applied successfully to predict lower shelf fracture toughness values of an A508 Cl.3 steel. However the capacity of this model to predict cleavage fracture toughness values with the same set of parameters when temperature increases, i.e. on the lower part of the transition region, has been questioned in the literature [4]. One underlying problem is that for the same temperature, extensive plasticity may develop in notched tensile specimens whereas it remains confined in deep-cracked geometries because of constraint conditions. On the other hand, the use of different temperatures for parameters calibration and toughness predictions raises concerns about the temperature effect on the parameters, which were assumed to be temperature independent in the original model.

In order to investigate the transferability of cleavage fracture parameters in the lower part of the transition region, a large experimental program was carried out on an A508 Cl.3 pressure vessel steel. The scope of the present study is to give some insights on the evolution of cleavage fracture mechanisms with increasing temperature for notched tensile and deep-cracked geometries. Secondly, based on a large number of notched specimens needed to deal with the statistical aspects, the parameters of the Beremin model have been calibrated at different temperatures.

\section{Experimental details and results}

\subsection{Material description and mechanical tests}

Tests were carried out on specimens cut from a forged nozzle shell of a pressurized water reactor of 220 mm thickness. The chemical composition of the material (French A508 Cl.3 steel) is given in Table 1. The material was received in heat treated conditions (two austenitizing treatments followed by water quenching 
and tempering, and a final stress relief treatment). The microstructure is essentially a tempered bainite with a room temperature yield strength $\sigma_{Y}=472 \mathrm{MPa}$ and an ultimate tensile strength, $\sigma_{U T S}=599 \mathrm{MPa}$. The typical microstructure is shown in Fig. 1.

\begin{tabular}{|c|c|c|c|c|c|c|c|c|c|c|}
\hline $\mathrm{C}$ & $\mathrm{S}$ & $\mathrm{P}$ & $\mathrm{Si}$ & $\mathrm{Mn}$ & $\mathrm{Ni}$ & $\mathrm{Cr}$ & $\mathrm{Mo}$ & $\mathrm{Cu}$ & $\mathrm{Co}$ & $\mathrm{V}$ \\
\hline 0.16 & 0.004 & 0.010 & 0.23 & 1.32 & 0.72 & 0.23 & 0.49 & 0.07 & 0.01 & 0.005 \\
\hline
\end{tabular}

Table 1: Chemical composition of A508 Cl.3 steel (wt.\%) from a forged nozzle shell.
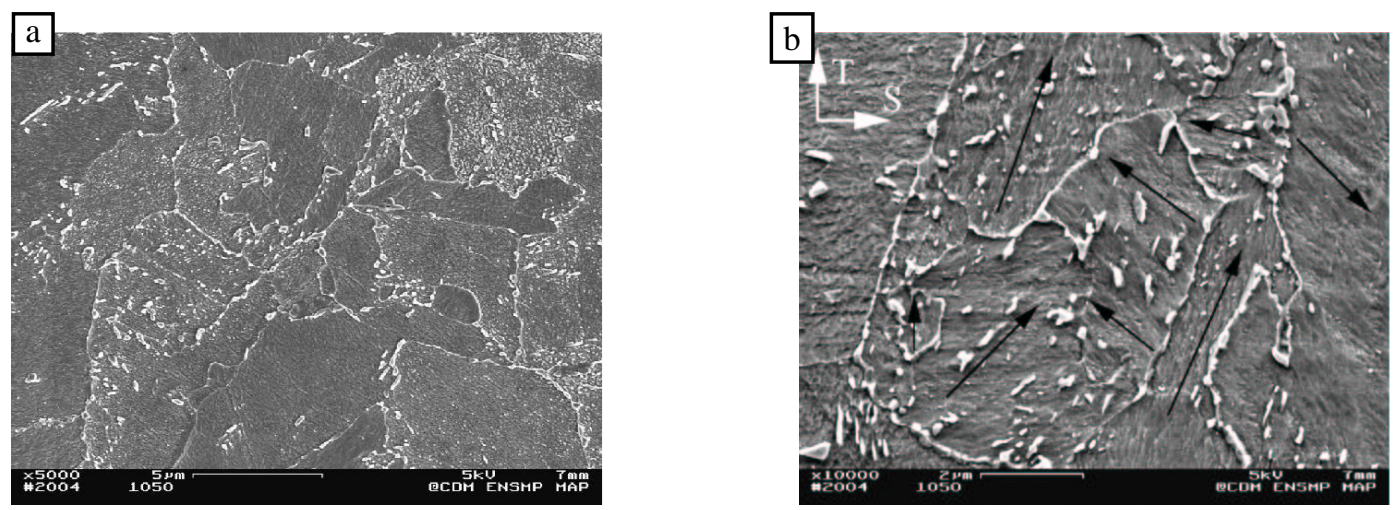

Figure 1: Microstructure of A508 Cl.3 steel. SEM micrography (electrolytically etched). Bainite laths orientations are indicated by arrows.

Conventional round tensile (RT) specimens of a nominal $5 \mathrm{~mm}$ diameter were used to determine the mechanical properties of the material. The tensile properties were determined for temperature between $-150^{\circ} \mathrm{C}$ and $-40^{\circ} \mathrm{C}$.

Axisymmetric notched tensile (NT) specimens defined by the ratio $R=\left(\rho / \Phi_{0}\right) * 10$ where $\rho$ is the radius of the notch and $\Phi_{0}(6 \mathrm{~mm})$ the minimum diameter were tested. Three notch geometries were used with $\rho=0.6,1.2$ and 2.4, i.e. $R=1,2$ and 4 . These tests were performed for temperatures between $-150^{\circ} \mathrm{C}$ and $-40^{\circ} \mathrm{C}$. During straining the actual minimum diameter $\Phi$ is continuously recorded. The mean fracture strain and stress were calculated as:

$\bar{\varepsilon}_{r}=2 \ln \frac{\Phi_{0}}{\Phi_{r}} \quad$ and $\quad \bar{\sigma}_{r}=\frac{4 \times F_{r}}{\pi \Phi_{r}^{2}}$

where $F_{r}$ is the load at fracture, $\Phi_{0}$ and $\Phi_{r}$ are the initial and fracture minimum diameters, respectively. In the case of the initiation of a ductile macro-crack at the center of the notch, failure (i.e. $F_{r}$ and $\Phi_{r}$ ) is defined at ductile crack initiation, i.e. at the onset of the sharp load drop.

Fracture mechanics specimens were tested over the temperature range between $-120^{\circ} \mathrm{C}$ and $-50^{\circ} \mathrm{C}$. Conventional CT type specimens of two different thicknesses $(B=12.5$ and $25 \mathrm{~mm})$ were used. These specimens were tested using the ASTM standards recommendations [5]. A clip gauge extensometer was used to continuously monitor the load line displacement, $\Delta L L$, during the tests.

T-S (long transverse-short transverse) compact tension (CT) and tensile (RT and NT) specimens were loaded along the long transverse $(T)$ orientation. All the specimens were machined from a layer located at $3 / 4$ thickness of the shell to minimize the material heterogeneity effects.

\subsection{Fractographic investigations}

The fracture surface of all the NT and CT specimens that failed by unstable fracture were examined in details using a SEM to locate the origin of cleavage fracture and determine the nature of particles or events initiating fracture. Localization on the fracture surface of the identified triggering sites was the second step in the 
fractographic study. Details about the methodology concerning the initiation site identification and its location measurements are given in [6,7]. When a particle was found at the initiation site, its nature was identified by $\mathrm{X}$-ray micro-analysis (when its size allowed it). For CT specimens, each site was quantified by the distance from the edge of the specimen, $x$, by the distance from the crack tip, $y$, and by the ductile crack length right at the triggering site location, $\delta a$, if any. It should be noticed that $\delta a$ is different from the average ductile crack length, $\overline{\Delta a}$, as defined by ASTM standards [5]. For NT specimens, the distance from the center of the fracture surface, $r$, was measured. All the measured distances used hereafter refer to the deformed configuration at fracture.

\subsection{Experimental results}

\subsubsection{Mechanical tests}

True stress-logarithmic strain curves (fig. 2a) reveal that this steel exhibits an upper and lower yield stress and a Lüders plateau. The yield stress, $\sigma_{Y}$, is then defined as the true stress value at the Lüders plateau. Evolutions of the yield stress, $\sigma_{Y}$ and of the ultimate stress, $\sigma_{U T S}$, with temperature are reported on fig. $2 \mathrm{~b}$.
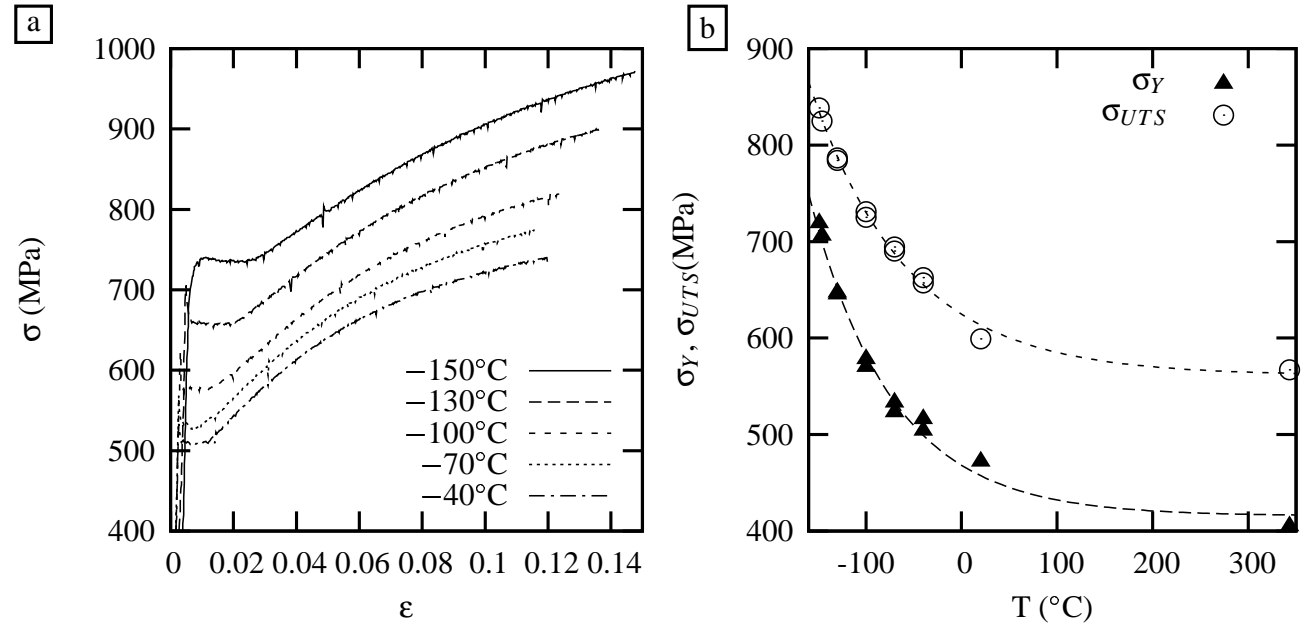

Figure 2: Tensile properties for the A508 Cl.3 studied steel. a) True stress-logarithmic strain curves b) Yield strength versus temperature.

Evolutions of the mean fracture stress, $\bar{\sigma}_{r}$, versus the mean fracture strain, $\bar{\varepsilon}_{r}$ at the different testing temperatures and as a function of the notch geometry are given in fig. 3. On each graph, filled black symbols are used when a ductile macrocrack has initiated at the center of the specimen before final failure by cleavage fracture. These tests were excluded from the database used for the Beremin parameters determination (see section 3).

For the precracked specimens, the minimum and maximum fracture toughness obtained at each testing temperature are reported in table 2 for both CT25 and CT12.5 specimens. In this table, $N_{\text {valid, }}$, is the number of valid tests following the ASTM E1921 standard, i.e. valid $K_{j c}$ fracture toughness values.

\subsubsection{Nature of the sites at the origin of cleavage}

The study of the nature of the sites at the origin of cleavage fracture has shown that large manganese sulfides $(\mathrm{MnS})$ inclusions were involved from low temperatures for the NT geometry, i.e. from $-150^{\circ} \mathrm{C}$ for the $\mathrm{NT}_{2}$ and $\mathrm{NT}_{4}$ and $-130^{\circ} \mathrm{C}$ for the $\mathrm{NT}_{1}$. They were always associated with large ductile voids. Depending on the temperature $\mathrm{MnS}$ inclusions which initiated cleavage were observed with different aspects (see fig. 4): (i) as an isolated large $\mathrm{MnS}$ particle surrounded by ductile damage which nucleated on a secondary population (ii) as a stringer which can be surrounded by ductile damage (fig. 4c) (iii) as a cluster with ductile damage linking the inclusion voids together (fig. 4a and b) (iv) as a set of several large inclusion voids very close to each others. In this paper, the generic expression MnS inclusions is used for all these different types. It is underlined that $\mathrm{MnS}$ inclusions may act directly or indirectly on cleavage initiation: cleavage initiates directly at the ductile 


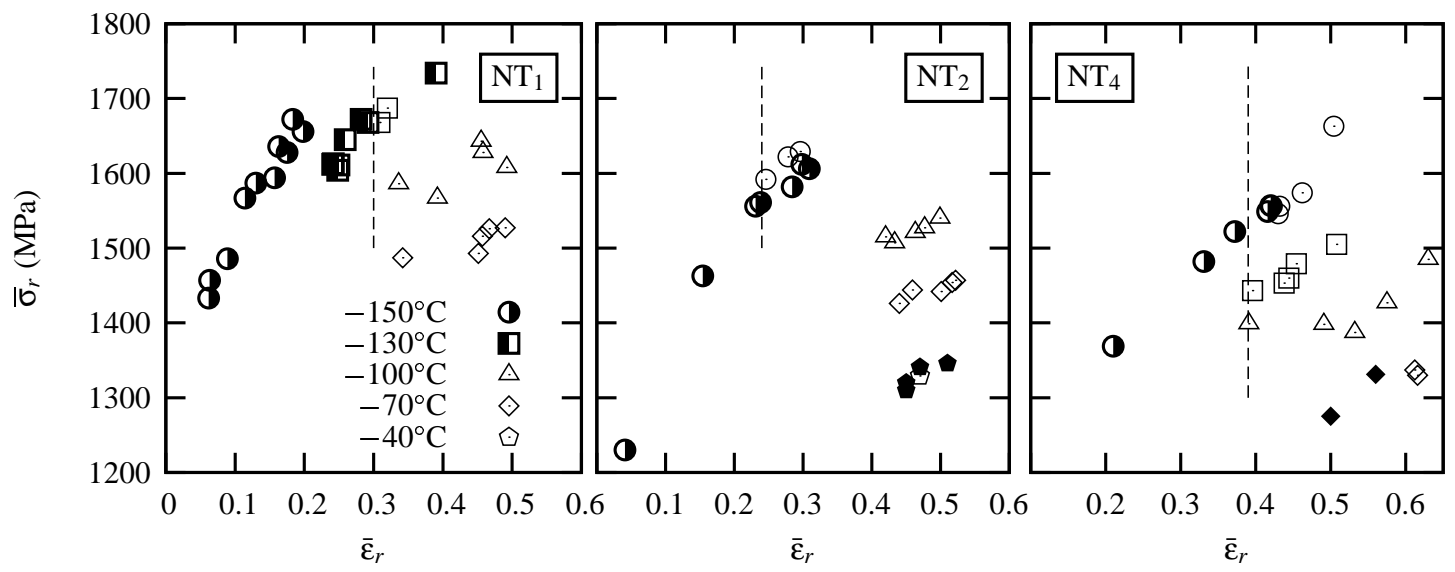

Figure 3: Evolution of the mean fracture stress versus strain with notch geometry and temperature (full-filled black symbols represent tests where a ductile macrocrack has initiated before cleavage fracture, half-filled symbols represents tests where large MnS inclusions were not found to be involved in the cleavage initiation).

\begin{tabular}{|l|c|c|c|c|c|}
\hline geometry & $\mathrm{T} /{ }^{\circ} \mathrm{C}$ & $\mathrm{N}$ & $K_{j c}^{\text {min }}--K_{j c}^{\max } / \mathrm{MPa} \sqrt{\mathrm{m}}$ & $K_{j}^{\text {lim }} / \mathrm{MPa} \sqrt{\mathrm{m}}$ & $\mathrm{N}_{\text {valid }}$ \\
\hline CT25 & -90 & 3 & $90.3-125.8$ & & 3 \\
CT25 & -80 & 6 & $64.7-167.6$ & & 6 \\
CT25 & -75 & 1 & 182.1 & & 1 \\
CT25 & -70 & 1 & 230.9 & & 1 \\
CT25 & -65 & 2 & $142.1-237.7$ & & 2 \\
CT25 & -50 & 11 & $102.5-413.1$ & 287 & 10 \\
CT12.5 & -120 & 6 & $86-136.2$ & & 6 \\
CT12.5 & -110 & 15 & $47.2-172$ & & 15 \\
CT12.5 & -70 & 15 & $92.5-254$ & 209 & 11 \\
CT12.5 & -50 & 15 & $171.9-442.7$ & 202 & 3 \\
\hline
\end{tabular}

Table 2: Experimental results on precracked specimens. $\mathrm{N}$ : number of tested specimens, $\mathrm{N}_{\text {valid }}$ : number of valid tests $\left(K_{j c} \leq K_{j}^{l i m}\right) . K_{j}^{l i m}=\sqrt{\frac{E \times b_{0} \times \sigma_{Y}}{\left(1-v^{2}\right) \times 30}}$ with $b_{0}$ the ligament length.

damage associated to the large ductile voids or cleavage may be triggered in the close surroundings of the MnS inclusions. In the first case, several cleavage initiation sites were often found all around the $\mathrm{MnS}$ inclusions zone. In the last case, it is inferred that $\mathrm{MnS}$ inclusions and associated ductile damage plays the role of stress raisers.

For each notch geometry, the domain where MnS inclusions were never found to be involved in the cleavage initiation corresponds to the left side of the dashed lines drawn on fig.3. Tests where MnS inclusions were found at the origin of cleavage are represented by open symbols on this figure. For $\mathrm{NT}_{1}$ specimens, it corresponds to tests with a mean fracture strain lower or equal to $30 \%$. For $\mathrm{NT}_{2}$ specimens, it corresponds to tests with a mean fracture strain lower or equal to $24 \%$ whereas a value of $39 \%$ was obtained for $\mathrm{NT}_{4}$ specimens. Also MnS inclusions were found as an unique type of triggering sites from $-130^{\circ} \mathrm{C}$ for $\mathrm{NT}_{4}$, from $-100^{\circ} \mathrm{C}$ for $\mathrm{NT}_{2}$ and from $-100^{\circ} \mathrm{C}$ for $\mathrm{NT}_{1}$ specimens.

For precracked specimens (CT25 and CT12.5), MnS inclusions associated to large voids were never found to be at the origin of the cleavage initiation in the temperature range $\left[-120 ;-50^{\circ} \mathrm{C}\right]$. Different types of triggering sites were found (see fig. 5) including small particle, grain boundary, microductility, local intergranular decohesion and intragranular facet. The nature of the sites was not found to correlate with the test temperature or with the thickness of the precracked specimens, at least in the investigated temperature range. 

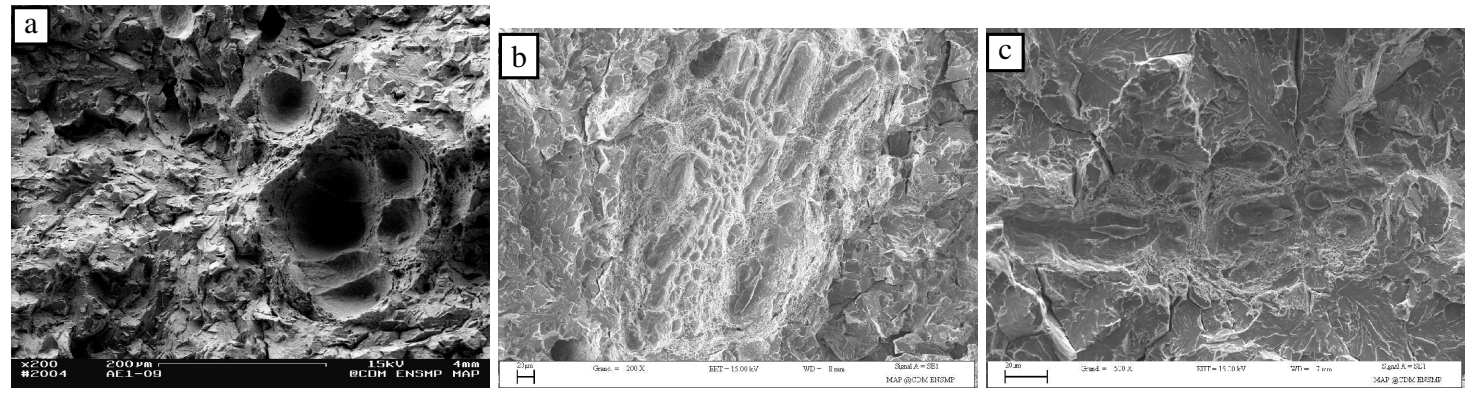

Figure 4: NT geometry. Different types of cleavage triggering sites where large $\mathrm{MnS}$ inclusions were found to be involved. a) $\mathrm{NT}_{1},-130^{\circ} \mathrm{C}$, b) $\mathrm{NT}_{1},-100^{\circ} \mathrm{C}$, c) $\mathrm{NT}_{2},-150^{\circ} \mathrm{C}$.
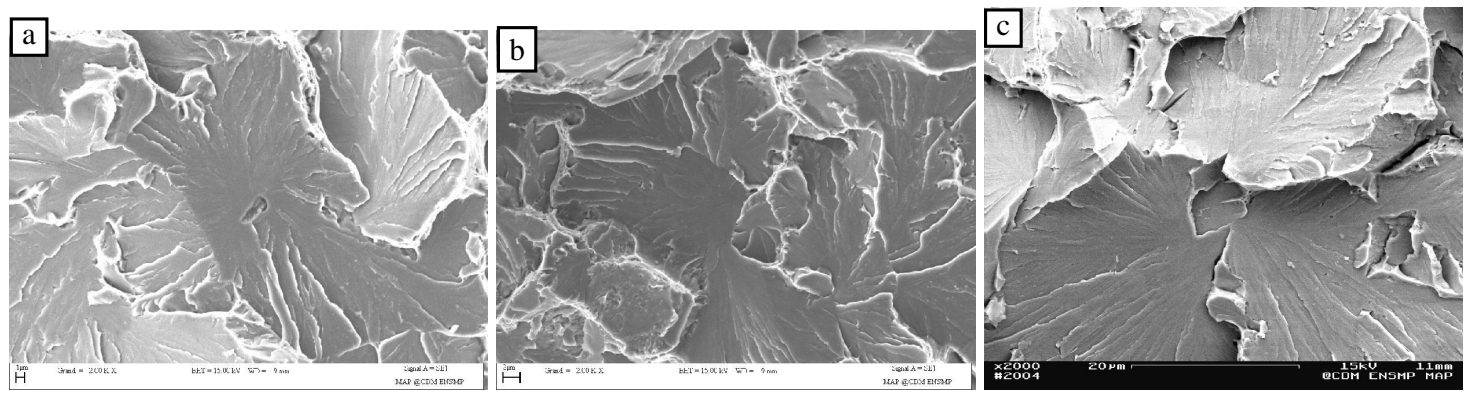

Figure 5: CT25 geometry. Different types of sites at the origin of cleavage a) Small rounded MnS inclusions, $-90^{\circ} \mathrm{C}$, b) Grain boundary, $-80^{\circ} \mathrm{C}$, c) grain boundary with the presence of a very small grain, $-75^{\circ} \mathrm{C}$.

\section{Finite element modeling, local mechanical and Beremin parameters determination}

Axisymmetric and 3D elasto-plastic finite element stress analysis were carried out on the NT and CT geometries employed in the present study.

As the Local Approach relies on a precise determination of the stress-strain fields in the fracture process zone of the different considered geometries, the following points have to be carefully checked: (i) the constitutive equation formalism and parameters have to be able to represent accurately the material behavior also for large strain levels met at the crack tip (for both SSY and LSY conditions) (ii) the size, the shape and the type of the finite elements have to be optimized to allow a good description of the crack tip blunting and of the mechanical gradients ahead of the crack tip, (iii) a sufficient number of elements through the thickness of the CT geometry has to be used to describe the out-of-plane stress gradient met in LSY conditions.

In order to fulfill the above requirements, the following strategy was adopted in this study: (i) the material behavior was described by a double nonlinear viscoplastic constitutive equation which has been already applied to describe successfully A508 $\mathrm{Cl} .3$ steel mechanical behavior $[8,9]$ (ii) load-displacement curves from both RT and NT tests were used to determine the material parameters related to hardening behavior. The isotropic derivation of a plastic criterion proposed recently [10] was used to obtain a very good agreement between experimental and numerical load-displacement curves for both RT and NT geometries. (iii) For the CT specimen mesh, the crack tip was treated as a very narrow notch with a semi-circular tip of radius $\delta / 2$. $\delta$ was chosen as a function of the experimental CTOD at fracture, $\delta_{f}$, following the criterion proposed by Mc Meeking [11]: $\delta<\delta_{f} / 10$ with $\delta_{f}=0.61 K_{j c}^{2} /\left(R_{p 0.2} E\right)$. Values of $\delta=4,10$ and $20 \mu \mathrm{m}$ were used in this study. (iv) 20 nodes 3D quadratic elements with full integration were used for CT specimens.

All the simulations presented here were conducted using the implicit finite element code ZeBuLon [12]. Finite strain effects were accounted for within a finite-strain Lagrangian framework. Figure 6 shows the FE mesh 
used to simulate the $\mathrm{NT}_{1}$ specimens and one of the $\mathrm{FE}$ meshes used for the $\mathrm{CT}$ specimen. A minimum mesh size of $50 \times 50 \mu \mathrm{m}^{2}$ was used for the NT meshes. For CT specimens 2 to $4 \mu \mathrm{m}$ long element were used at the crack tip depending on the $\delta_{f}$ value.
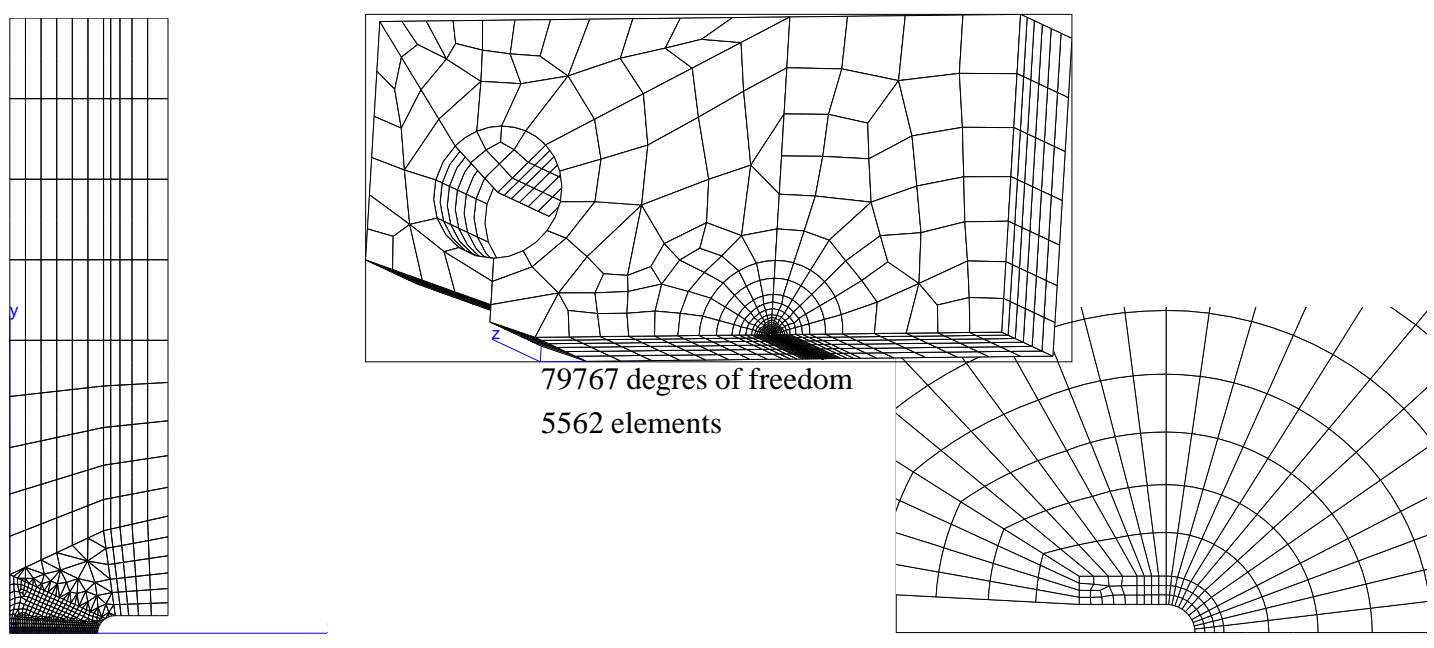

Figure 6: Meshes of the $\mathrm{NT}_{1}$ and $\mathrm{CT} 25$ geometries used in the FE simulations. The root radius of the crack tip shown is $2 \mu \mathrm{m}$.

\subsection{Local mechanical parameters determination}

Based on the location of the initiation site in the deformed NT and CT geometries, the local stress and strain at fracture were evaluated from finite element calculations. The maximum principal stress and the cumulated plastic strain were selected as representative local values of the loading at failure. For simplification, it was assumed that, for both NT and CT geometries, the initiation points lay on the mid-plane. As fracture occurs after large scale yielding for some of the CT25 tests and for most of the CT12.5 tests, 3D FE analysis have been carried out and the stress and strain values were computed at the gauss point the closest of the initiation site in the thickness direction. Before computing the maximal principal stress, $\sigma_{I}$, the stress tensor is smoothed in order to obtain a constant stress tensor trace in each element (see [9]). This procedure allows us to smooth the pressure variations caused by the quasi-incompressibility of the material while preserving a more accurate stress profile.

\subsection{Beremin parameters determination}

Model parameters $\left(\sigma_{u}\right.$ and $m$ ) (eq. 2$)$ were first identified using the NT specimens.

The parameters were identified using an automatic identification procedure which minimizes the quadratic differences between the theoretical and experimental failure probabilities. For a given set of model parameters, the Weibull stress at experimental failure and the associated theoretical failure probability (eq. 2) were computed for each sample. Using the same Weibull stress, the different tests were ordered (increasing Weibull stress) to compute the experimental failure probability as: $P_{r}^{i}=\left(i-\frac{1}{2}\right) / N$ where $N$ is the total number of specimens and $i=1 \ldots N$ the rank of the specimen. The reference volume, $V_{0}$, was taken equal to $0.001 \mathrm{~mm}^{3}$.

$\sigma_{w}=\left[\int_{V} \tilde{\sigma}_{I p}^{m} \frac{d V}{V_{0}}\right]^{1 / m} \quad P_{R}=1-\exp \left[-\left(\frac{\sigma_{w}}{\sigma_{u}}\right)^{m}\right]$

\section{Results}

\subsection{Local stresses and strains versus temperature and geometries}

Among the whole set of the NT specimens, only those which have led to cleavage fracture before the initiation of a ductile macrocrack were considered in this study, i.e. 64 specimens. Among these 64 specimens, 42 
have been observed to date to locate the cleavage initiation sites. All the 25 CT25 specimens and 39/61 CT12.5 specimens have been observed. The evolution of the local fracture stress as a function of geometry and temperature is reported on fig. 7. On fig. 7, the NT tests which have a mean fracture strain lower than the minimum one which corresponds to $\mathrm{MnS}$ inclusions involvement in the cleavage process initiation for each notch geometry (see fig. 3) are represented by filled black symbols. From figure 7, it is shown that: (i) as far as the MnS inclusions are not involved in the cleavage initiation process the mean local fracture stress obtained on NT specimens is very close to the one obtained on CT 25 geometry, (ii) in the temperature range $\left[-90^{\circ} \mathrm{C} ;-50^{\circ} \mathrm{C}\right]$, the mean local fracture stress value is identical for CT25 and CT12.5 specimens, (iii) at $-120^{\circ} \mathrm{C}$ and $-110^{\circ} \mathrm{C}$, the mean $\sigma_{f}$ value obtained from CT12.5 is slightly higher than the one obtained in the range $\left[-90^{\circ} \mathrm{C} ;-50^{\circ} \mathrm{C}\right]$ (this result is still under investigation), (iv) a threshold value of $1400 \mathrm{MPa}$ is obtained for all the geometries in the whole temperature range investigated.

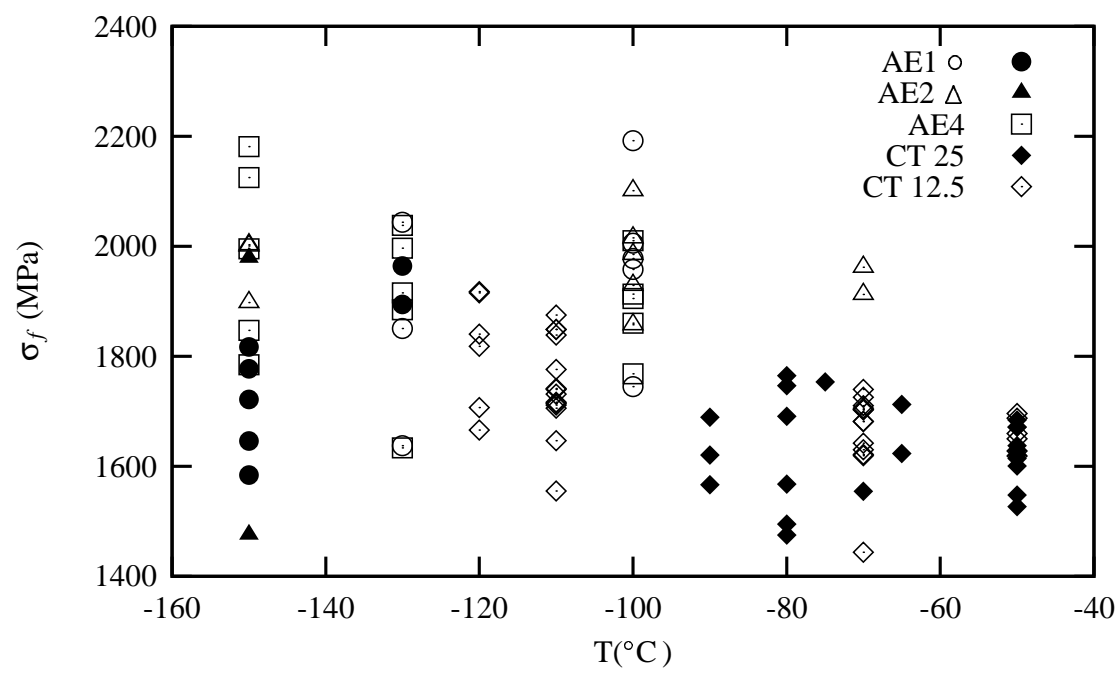

Figure 7: Local fracture stresses calculated at the cleavage initiation sites for the NT and CT tests.

\subsection{Beremin parameters determined from notched geometry}

As previously stated one of the goals of this study was to investigate the potential dependence of the parameters $\sigma_{u}$ and $m$ on temperature or with geometry. Firstly, $m$ and $\sigma_{u}$ were determined for each temperature and geometry when a sufficient number (at least 10) of experimental results was available, i.e. at $-150^{\circ} \mathrm{C}$ for the three notch geometries and at $-130^{\circ} \mathrm{C}$ for the $\mathrm{NT}_{1}$ geometry. Secondly $m$ and $\sigma_{u}$ were determined for a given temperature mixing the three different NT geometries. Finally all the experimental results ( 75 tests) were considered to determine the Beremin parameters. The $m$ and $\sigma_{u}$ values are given in Table 3. Weibull stress distributions as a function of temperature are given in figure 8 .

\begin{tabular}{|l|c|c|c|c|}
\hline geometry & $\mathrm{T} /{ }^{\circ} \mathrm{C}$ & $\mathrm{N}$ & $m$ & $\sigma_{u} / \mathrm{MPa}$ \\
\hline $\mathrm{NT}_{1}$ & -150 & 10 & 19.7 & 2884 \\
$\mathrm{NT}_{1}$ & -130 & 10 & 26.2 & 2719 \\
$\mathrm{NT}_{2}$ & -150 & 10 & 28.6 & 2683 \\
$\mathrm{NT}_{4}$ & -150 & 10 & 38.6 & 2476 \\
\hline \hline All NT & -150 & 30 & 20.8 & 2956 \\
All NT & -130 & 15 & 21.7 & 2893 \\
All NT & -100 & 15 & 19.9 & 3014 \\
All NT & -70 & 13 & 19.6 & 2919 \\
\hline \hline All NT & All T & 75 & 20.1 & 2976 \\
\hline
\end{tabular}

Table 3: Beremin parameters determined on notched specimens. $\mathrm{N}$ : number of specimens. 

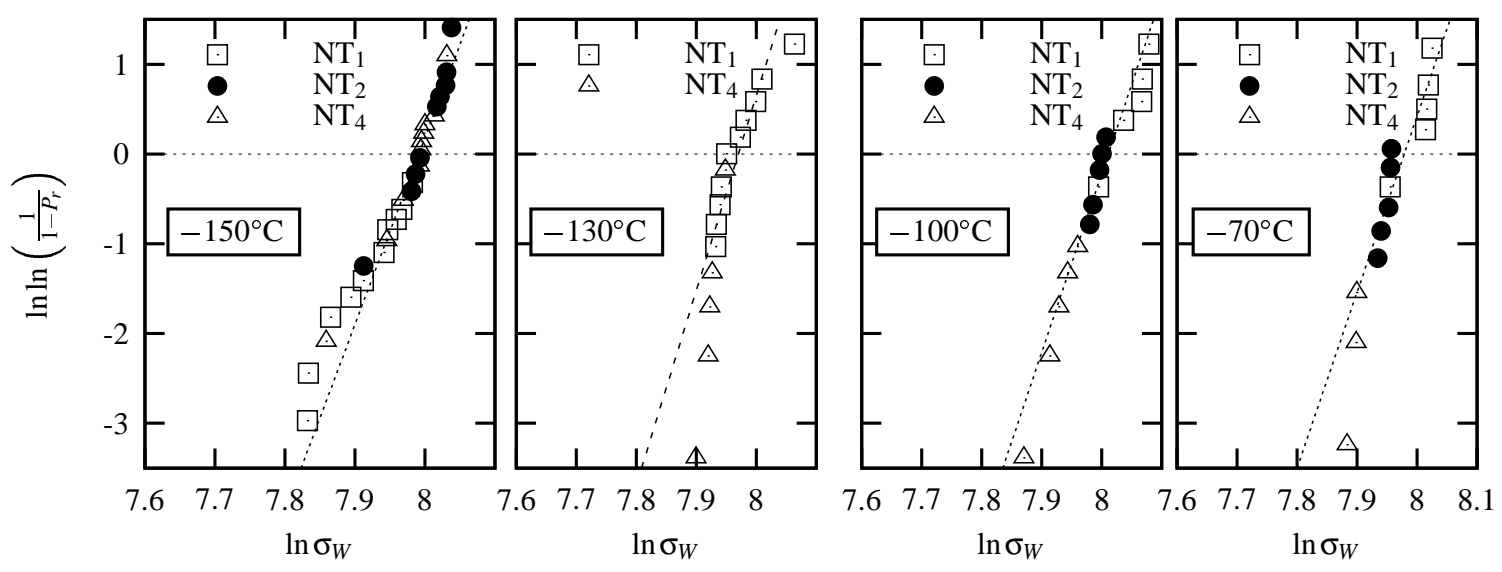

Figure 8: Weibull stress distribution of NT specimens at four temperatures.

In table 3 it is shown that, when for a given temperature all the three notch geometries are mixed, the values of $m$ and $\sigma_{u}$ are independent of the test temperature in the range $\left[-150^{\circ} \mathrm{C} ;-70^{\circ} \mathrm{C}\right]$. Also the $m$ and $\sigma_{u}$ values found with all the tested specimens (75) are similar to those found at each temperature when the three notch geometries are considered (see fig. 9). From the whole set of NT tests, the values $m=20.1$ and $\sigma_{u}=2976 \mathrm{MPa}$ are obtained assuming that $V_{0}=0.001 \mathrm{~mm}^{3}$. Considering the $m$ and $\sigma_{u}$ values for one notched geometry and one temperature, some variations are obtained. From SEM observations, these variations may be explained by the role of the MnS inclusions in the cleavage initiation process.
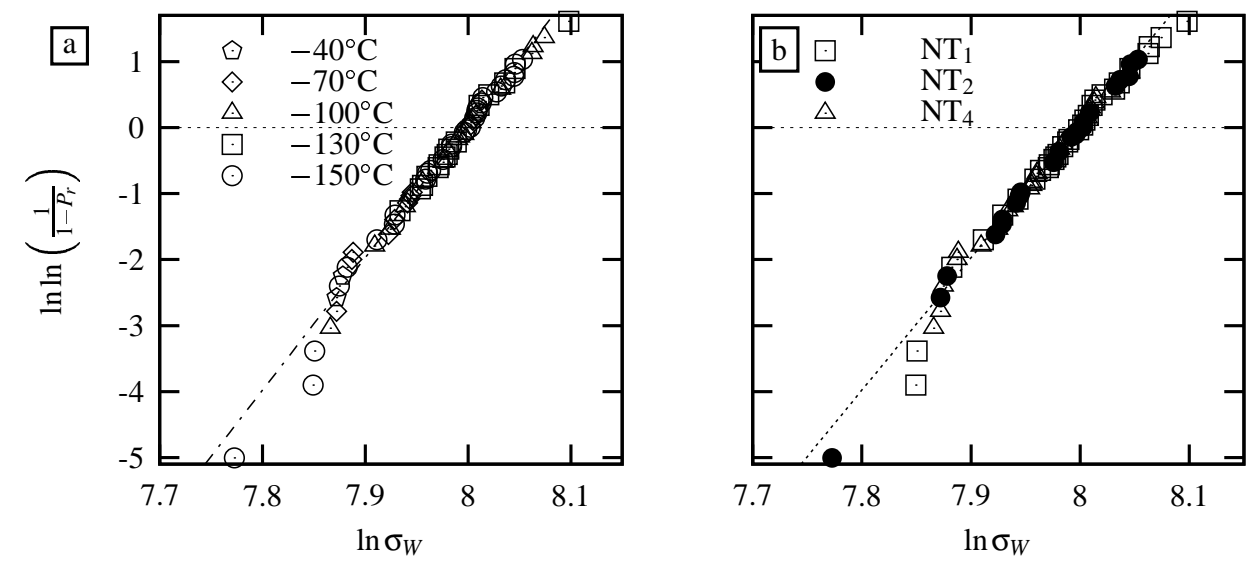

Figure 9: Weibull stress distribution of all the NT specimens. a) Results ranked by temperatures b)results ranked by notch geometry.

\subsection{Prediction of the fracture toughness}

The values of $m$ and $\sigma_{u}$ obtained from the whole set of NT tests, i.e. $m=20.1$ and $\sigma_{u}=2976$, have been applied to predict the load line displacement at fracture $\left(\Delta L L_{c}\right)$ for the CT25 specimens. To obtain a good agreement between experimental and numerical load- $\Delta L L$ curves, a von Mises plasticity criterion has been used to simulate the CT25 specimens. At each temperature, the fracture probability, $P_{R}$, was computed. The results are reported on figure 10. Numerical convergence problems have been encountered due to the large blunting of the crack tip for high loading levels so that for each temperature the value of $P_{R}$ at the highest $\Delta L L$ computed is reported on the graph. It is shown that the $5 \%$ fracture probability predictions are in good agreement with the experimental data. At low temperature $\left(-90^{\circ} \mathrm{C}\right.$ and $\left.-80^{\circ} \mathrm{C}\right)$, the predictions are found to 
underestimate the experimental data, i.e. the calculated failure probability value is too low as compared to the experimental results. A better agreement is obtained at $-50^{\circ} \mathrm{C}$ between the model and the experimental data.

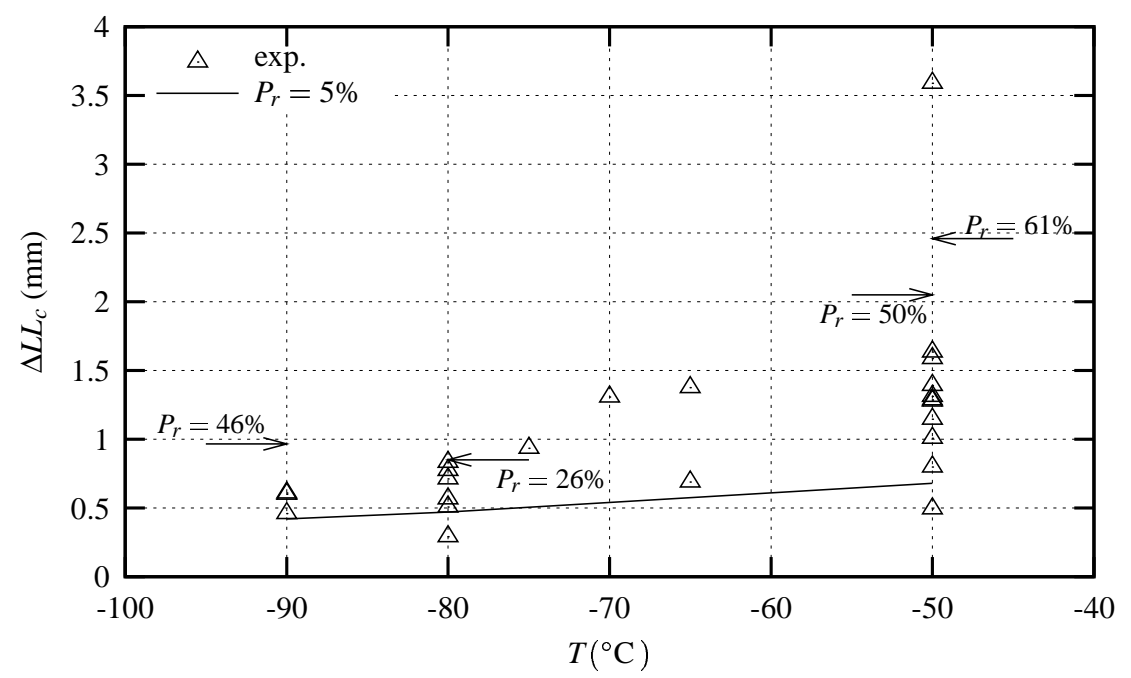

Figure 10: Load Line displacement at fracture predictions for CT25 specimens using local approach parameters determined on notched tensile specimens.

\section{Conclusions and further work}

In this paper, the cleavage fracture behavior of tensile notched and precracked specimens of a French A508 Cl.3 RPV steel in the lower up to mid transition range has been investigated. Based on a large experimental database, SEM observations and finite element calculations, the cleavage fracture mechanisms, mechanical stress and strain conditions and the transferability of Beremin parameters have been studied. The following conclusions may be drawn concerning the steel investigated:

1. In the range $\left[-120^{\circ} \mathrm{C} ;-50^{\circ} \mathrm{C}\right]$, large $\mathrm{MnS}$ inclusions were never found at the main cleavage initiation site in precracked specimens. Small $(\Phi<5 \mu \mathrm{m})$ rounded $\mathrm{MnS}$ inclusions without ductile damage can be found at the origin of the cleavage initiation among other types of sites: grain boundaries, local intergranular facet, small island of ductility, TiN or Fe3C particles. In precracked geometry, the type of sites were not found to be dependent on temperature.

2. For the three studied notch geometries $\left(\mathrm{NT}_{1}, \mathrm{NT}_{2}\right.$ and $\mathrm{NT}_{4}$ ), large $\mathrm{MnS}$ inclusions were found to act in the cleavage initiation process when specimens were tested at relatively elevated temperature. The large $\mathrm{MnS}$ inclusions were never found at the cleavage triggering site for mean fracture strains lower than $30 \%$ for $\mathrm{NT}_{1}, 24 \%$ for $\mathrm{NT} 2$ and $39 \%$ for $\mathrm{NT}_{4}$. This means that to ensure that a change of the nature of potential defects for cleavage will not appear, only NT tests with a mean fracture strain lower or equal to $\sim 25 \%$ have to be considered.

3. It is found that when the NT tests giving rise to cleavage initiation from large MnS inclusions are excluded the mean local cleavage is similar in notched and cracked specimens, and is independent of temperature over the temperature range $-150^{\circ} \mathrm{C} ;-70^{\circ} \mathrm{C}$.

4. A threshold of $1400 \mathrm{MPa}$ for the local cleavage stress was found for all the geometries and temperature tested.

5. Mixing the results obtained on different notched geometries, the Beremin parameters are shown to be approximately constant with temperature. Considering separately each notch geometry, some variations have been obtained with temperature. These variations have to be further investigated in increasing the number of specimens tested at each temperature. However it is inferred that they are linked to a change in the role of $\mathrm{MnS}$ inclusions in the cleavage initiation process. 
6. Considering the Beremin parameters obtained from the whole set of NT specimens, model predictions were found to slightly underestimate the cleavage fracture toughness of CT25 specimens, especially at low temperature $\left(-90^{\circ} \mathrm{C}\right)$. A better agreement was found at higher temperature $\left(-50^{\circ} \mathrm{C}\right)$.

\section{Acknowledgments}

Financial support from Direction de la Sureté des Installations nucléaires (DSIN) and Electricité de France $($ EdF) is acknowledged.

References

1 Burstow, M., Beardsmore, D., Howard, I. and Lidbury, D., Int. J. Pressure Vessel Piping, 80, 775-785, 2003.

2 Pineau, A., In: Comprehensive Structural Integrity, Ainsworth, R. and Schwalbe, K.-H. (Eds.), volume 7. Elsevier Pergamon, 177-225, 2003.

3 Beremin, F., Met. Trans., 14A, 2277-2287, 1983.

4 Wiesner, C. and Goldthorpe, M., In: 1st european mechanics of materials conference on local approach to fracture, Journal de Physique IV, Les Editions de Physique (Ed.), volume 6. Fontainebleau, 9-11 Septembre, C6-295-C6-304, 1996.

5 ASTM-E1820 Standard test method for measurement of fracture toughness. ASTM, Philadelphia,USA, 2001.

6 Tanguy, B., Besson, J., Piques, R. and Pineau, A., Engng Fract Mech, 72/1, 49-72, 2005.

7 Bouchet, C., Arnoldi, F., Besson, J., Degallaix, S., Denner, V., Desplanques, Y., Diard, O., Espinasse, G., Forget, P., Hausild, P., Maire, E., I., N., Rabeau, V., Tanguy, B. and Verdu, C., In: 11 th International Conference on Fracture. Turin, Italy, 2005.

8 Tanguy, B., Piques, R., Laiarinandrasana, L. and Pineau, A., In: EUROMAT 2000, Advances in Mechanical Behaviour. Plasticity and Damage, Miannay, D., Costa, P., François, D. and Pineau, A. (Eds.). Elsevier, 499-504, 2000.

9 Tanguy, B., Besson, J., Piques, R. and Pineau, A., Engng Fract Mech, 72/3, 413-434, 2005.

10 Bron, F. and Besson, J., Int. J. of Plasticity, 20, 937-963, 2004.

11 McMeeking, R., J. Mech. Phys. Solids, 25, 357-381, 1977.

12 Besson, J. and Foerch, R., Computer Methods in Applied Mechanics and Engineering, 142, 165-187, 1997. 